\title{
Counsellors Practices of Ethnic and Religious Factors in Secondary Schools
}

\author{
Mohamed Sharif Mustaffa, ${ }^{a^{*}}$ Amalia Madihie, ${ }^{a}$ Prashanth Talwar Yadar ${ }^{a}$ and Aqeel Khan ${ }^{b}$ \\ ${ }^{a}$ FSKPM, Universiti Malaysia Sarawak, Malaysia. \\ ${ }^{b}$ Faculty of Education, Universiti Teknologi Malaysia \\ Corresponding Author E-mail: *msharif@unimas.my.
}

This study explored the everyday experiences of Muslims' school counsellors as they encounter and negotiate ethnic and religious contextual factors in their practices in Malaysian secondary schools. Specially, the study also explored how Muslims' school counsellors adapt their generic, Western-oriented training models and practices to the needs of everyday practice in Malaysian society. In particular, the researcher investigated the influence of multi-ethnicity of students and diversity of religion in their counselling contexts. A major difficulty is that much of the literature appears to be general, theoretical or ideological, rather than based on the actual experiences of practicing counsellors in the countries concerned. In order to focus on experience of counsellors, a qualitative case study approach was used. The researcher examined the practice of eleven Muslims' school counsellors in the district of Muar, using a combination of interviews, observation and document analysis. The data suggested that counsellors actively adapt their training models in various ways in relation to the two factors studied. Counsellors perceived a considerable gap between what they learned in their university training course and the knowledge and skills required for actual practice.

Keywords: Counsellor, Ethnic, Religious Factors, Secondary School, Malaysia

\section{INTRODUCTION}

Lemberger ${ }^{1}$ developed a humanistic theory for school counselling, advocating studentwithin-environment, to better meet needs in a culturally sensitive manner. The advocating student-within-environment theory allows school counselors to consider their environment, but to use their individuality within the concepts of the environment. School counsellors in Malaysia reported that lack of training in counselling strategies which are relevant and applicable to the realities of a school setting. Multicultural Counselling Theory focuses on addressing cultural differences in counselling to ensure that clients are able to make individual choices that are appropriate for them, not the counselor's views or society's views in particular. ${ }^{2}$ Indeed whether the particular theory is psychodynamic, existential-humanistic, or behavioural in orientation. Ivey, Ivey, and Simek-Morgan ${ }^{3}$ indicate that they share certain common components of Western culture in their values and beliefs. These particular values and beliefs have influenced the formation and practice of counselling. Much of the work in areas such as multicultural counselling has been theoretical or ideological, or has focused on generalized outcomes and effects on clients. Hence, the objectives of this study were to explore (1) the everyday experiences of Muslims' school counsellors as they encounter and negotiate ethnic and religious contextual factors in their practices in the Malaysian secondary schools, and (2) Muslim school counsellors adapt their generic, Western-oriented training models and practices to the needs of everyday practice in Malaysian society. 